\title{
Energy-efficient data dissemination strategy for roadside infrastructure in VCPS
}

\author{
Jin Qian ${ }^{*}$, Tao Jing, Yan Huo, Hui Li and Zhen Li
}

\begin{abstract}
In vehicular cyber-physical system (VCPS), beaconing mechanism is commonly deployed for the solar-powered roadside infrastructure to detect available passing-by vehicles for data dissemination. The traditional beaconing mechanism would cause a higher energy consumption due to the periodical beaconing procedure. To tackle this problem, we propose a new data dissemination strategy for roadside infrastructures by adjusting the beaconing interval to reduce the energy consumption. We model the beaconing procedure as a Markov model by observing the periodical beaconing results and then using the modeling result to obtain the relationship between the beaconing interval and the expectation of the single-vehicle discovery time. Then, with the average data transmission rate requirement, we can calculate the maximum beaconing interval. We also introduce a satisfaction degree based on the Sigmoid function to combine the decreased energy consumption and the decreased data rate. The satisfaction degree function allows the roadside infrastructure to obtain the optimal beaconing interval based on the required quality of service. The analysis of the results show that careful tuning of key parameters leads to improved energy efficiency and increased data rate of the data dissemination strategy.
\end{abstract}

Keywords: VCPS, Beaconing interval, Markov model

\section{Introduction}

Cyber-physical system (CPS) is a complex system with intricate interplay between the physical world and the cyber world [1-4]. Vehicular cyber-physical system (VCPS) is a typical CPS applied in the intelligent transportation field. It has attracted a significant amount of interests in the past few decades [5-8]. VCPS holds great potential in enhancing vehicle safety and improving traffic efficiency. It also can make a further contribution to passengers' comfort by providing infotainment services.

In VCPS, roadside infrastructures play an important role. They are usually deployed in highways or road intersections and can communicate with passing-by vehicles through dedicated short-range communications (DSRC) technologies. According to the difference of the structure and the function, roadside infrastructures could generally be divided into two types. One is with a large communication coverage and high processing capability [9-11]. They are used as wireless gateway nodes that interconnect

${ }^{*}$ Correspondence: qianjin@bjtu.edu.cn

School of Electronics and Information Engineering, Beijing Jiaotong University, Haidian, 100044 Beijing, China vehicular ad hoc networks and a wired network (i.e., the Internet), providing Internet connectivity to the vehicles. Also, they offer the real-time traffic status to the vehicles. The other kind is the simple structure roadside infrastructure just with the capability to disseminate data [12]. It disseminates the data stored in the infrastructure to the passing-by vehicles periodically. Generally, these data are the advertising messages. Due to the characteristics of the convenient installation and the relatively low cost of these simple structure roadside infrastructures, they have been widely used in VCPS. With the massive uses of them, the information content diversity of the whole network will be enriched leading to a further improvement of the VCPS. The research objects of this paper are these simple structure roadside infrastructures.

These simple structure roadside infrastructures are massively deployed in locations like the highway. Normally, they use the solar as power input due to the unavailability or excess expense of wired electrical power. While the provisioning cost of a solar-powered roadside infrastructure is a strong function of its average energy consumption. According to the analysis in [13] which is 
a part of the US Department of Transportation's Vehicle Infrastructure Integration Initiative, a breakdown of the deployment costs also found that over $63 \%$ of these roadside infrastructure costs would be consumed by solar energy provisioning, e.g., solar panels, batteries, and their associated electronics. So, it is important to reduce the energy consumption of the simple structure roadside infrastructures.

The energy consumption is mainly composed of two parts: the energy consumed in the wireless communication and the energy consumed in the information processing and computing. The radio is the most energyconsuming component, taking nearly 30 times the energy of the microcontroller unit used in the simple structure roadside infrastructures [14]. Therefore, to reduce the energy consumption, the key point is to improve the energy efficiency of the wireless communication.

For these simple structure roadside infrastructures, the basic mechanism of the traditional data dissemination strategy is beaconing $[15,16]$. Beacon packets are periodically and locally broadcasted by a roadside infrastructure to announce its current status and detect whether there are suitable vehicles to disseminate the data to. One vehicle in the communication range that successfully receives the beacon packet needs to reply an acknowledgment (ACK) packet. After receiving the ACK packet, the roadside infrastructure can get the status of the vehicle. Then, it can decide whether to start the data transmission process or not based on the status of the vehicle and the specific data content. In our research, we find that if the vehicle density is relatively low around the location where the roadside infrastructure is deployed, due to the limited number of the passing-by vehicles, just a few numbers of the beacon packets get a reply. Most of the beacon packets are ineffective. Although the beacon packet is small, larger numbers of ineffective beacon packets cause a high energy waste.

In this paper, we design an energy-efficient data dissemination strategy for roadside infrastructures by adjusting the beaconing interval. We design two phases for the new data dissemination strategy. The first is the modeling phase. The second is the applying phase. We model the beaconing procedure as a Markov model by observing the periodical beaconing results in the modeling phase and then use the modeling result in choosing a new beaconing interval in the applying phase. With the average data transmission rate requirement, we can calculate the maximum beaconing interval. By using the Sigmoid function, we can obtain the optimal beaconing interval based on the satisfaction degree. Through the proposed strategy, we can increase the effectiveness of the beaconing packets and improve the energy efficiency of the data dissemination strategy. Our main contributions are summarized as follows:
1. According to the Markov property of the presence of the passing-by vehicles, we present the state transition probabilities for the Markov model of the beaconing results by using the maximum likelihood method. Meanwhile, we calculate the duration of the modeling phase within which the modeling can guarantee a certain accuracy based on the central limit theorem.

2. We obtain the relationship between the beaconing interval and the expectation of the single-vehicle discovery time based on the state transition probabilities. The single-vehicle discovery time means the time interval between the adjacent two times when the roadside infrastructure discovery one vehicle. With the average data transmission rate requirement which is related to the single-vehicle discovery time, we can calculate the maximum beaconing interval.

3. We introduce a satisfaction degree based on the Sigmoid function to combine the decreased energy consumption and the decreased data rate effectively. If we want to choose a smaller beaconing interval than the maximum beaconing interval and obtain a shorter single-vehicle discovery time, we can use the satisfaction degree function to obtain the optimal beaconing interval which can balance the tradeoff between energy consumption and the average data transmission rate.

4. We demonstrate that the proposed data dissemination strategy can significantly reduce the energy consumption while satisfying the service quality requirement. The impact of the system parameters on the data dissemination strategy is investigated via extensive simulation study. The analysis of the results show that careful tuning of key parameters leads to improved energy efficiency and increased data rate of the data dissemination strategy.

The rest of the paper is organized as follows. Related work is outlined in Section 2. Our system model and the problem definition are described in Section 3. In Section 4, we describe the detail of the modeling phase. The applying phase is described in Section 5. The whole beaconing interval optimization process is given in Section 6. In Section 7, we make a numerical analysis of our strategy. The conclusion is finalized in Section 8.

\section{Related work}

For the roadside infrastructures, energy conservation in the data dissemination strategy guarantees the efficient usage of energy, which makes great contribution to the commercial application of VCPS. Recently, many researches have been made to improve energy efficiency. In [17], by using a mixed-integer linear programming 
Table 1 The beaconing results of the four scenarios

\begin{tabular}{lcccc}
\hline & Scenario 1 & Scenario 2 & Scenario 3 & Scenario 4 \\
\hline Direction 1 & 0 & 0 & 1 & 1 \\
Direction 2 & 0 & 1 & 0 & 1 \\
\hline
\end{tabular}

optimization method, the author proposes a data dissemination strategy with the ability of satisfying the communication requirements while minimizing the energy consumption. In the highway scenario, the location of vehicles passing through the roadside infrastructure radio coverage area can be predicted with a high degree of accuracy. Hammad et al. [18] use this information to reduce the energy cost of the downlink infrastructure-to-vehicle data dissemination. In [19], to obtain a energy-efficient data dissemination strategy, authors classify the energy efficiency problem into three categories and formulate them as a three-step optimization problem. In [20] and [21], two different on/off sleep data dissemination strategies are proposed. In these strategies, if there is no request received from vehicles, the roadside infrastructure works alternately between ON and OFF in a periodical manner to reduce the energy consumption. In [22], a variable bit rate transmission is used to increase the energy efficiency and the roadside infrastructures adapt its bit rate to accommodate different channel path loss conditions. However, all the above studies are still based on the periodically beaconing strategy. The problem of how to optimize the beaconing interval to reduce the energy consumption is still a whitespace in these papers.

\section{Problem definition}

In this paper, we study about the energy efficiency of the simple structure roadside infrastructures which are deployed in locations like the highway. Generally, two roadside infrastructures are deployed far away from each other and there is no connection between them. So, we just need to consider one roadside infrastructure on the highway. For one simple structure roadside infrastructure, it does not need to disseminate the data to every passing-by vehicles. It just needs to disseminate the data to parts of the passing-by vehicles and ensures a certain average data transmission rate.

In the traditional data dissemination strategy, the beaconing process is similar to a detecting process. The roadside infrastructure sends a beacon packet on the control channel to detect whether there is a vehicle that can receive the data or not. One passing-by vehicle that successfully receives the beacon packet needs to reply an ACK packet which includes the vehicle status like moving direction, velocity, and location. Generally, the data dissemination strategy is applied separately for the two traffic flow directions since most of the data contents are related to the traffic flow directions. To help understanding, four groups of beaconing results are given in Table 1 corresponding to the four scenarios in Fig. 1. If the roadside infrastructure successfully detects one or more vehicles, we denote the beaconing result as 1 ; otherwise, we denote it as 0 . Need to note that, in the traditional data dissemination strategy, the duration of the beaconing period can guarantee that one vehicle could be detected at most one time when it passes through the communication range of the roadside infrastructure. If one vehicle repeatedly receives the same beacon packet, it only replies the ACK message once. For convenience, we just consider one traffic flow direction in the following sections since the beaconing processes in both directions are separate and similar. The results for one direction can be readily generalized to the case of two directions.

The periodical beaconing process in traditional data dissemination strategy is shown in Fig. 2. In $k$ th period, the beaconing result is denoted as a Boolean variable $R_{k}$. $\mathbf{R}=\left\{R_{1}, R_{2}, \cdots, R_{N}\right\}$ represents the beaconing results of the whole beaconing process. $N$ is the number of the beaconing periods and also is the number of the beaconing results. As we mentioned in the previous section, the number of passing-by vehicles is limited in the low-vehicle density scene. Large numbers of continuous zeroes appear in $\mathbf{R}$. From the energy point of view, beaconing in this zero value time is meaningless. The data dissemination strategy
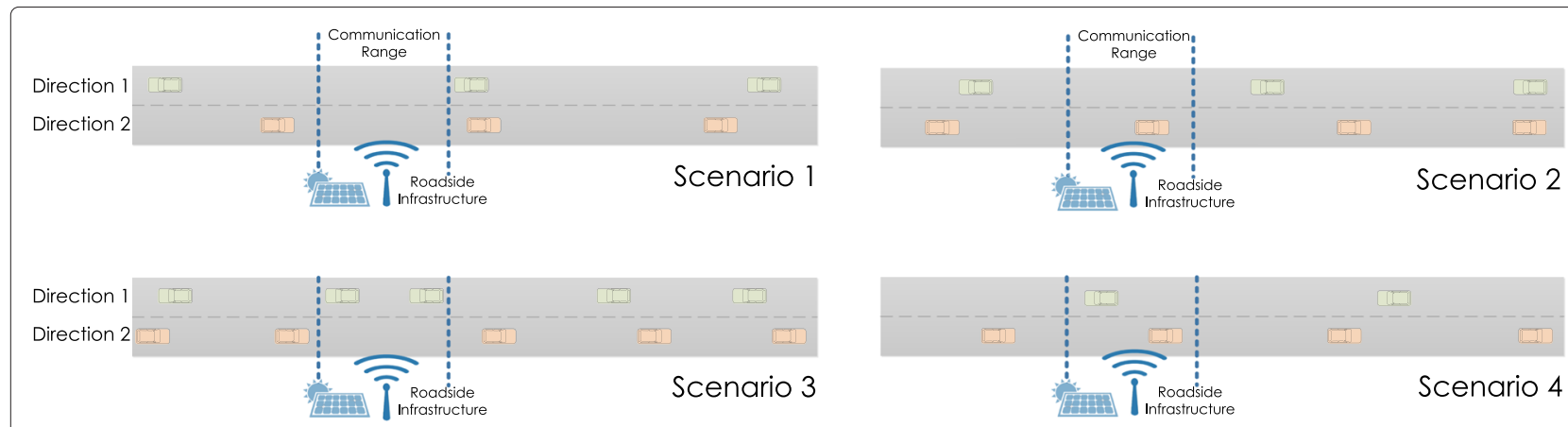

Fig. 1 Four beaconing scenarios in the data dissemination strategy

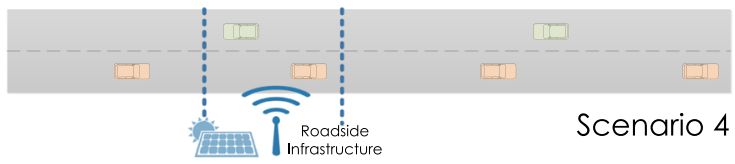




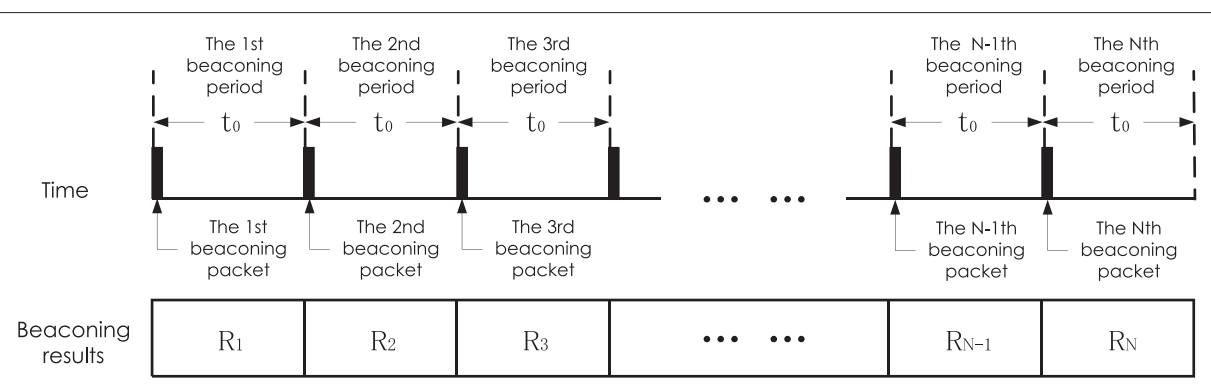

Fig. 2 The periodical beaconing process in the traditional data dissemination strategy

will be more energy efficient if the roadside infrastructure broadcasts the beacon packets with a certain interval instead of broadcasting them in each period. This is the core idea of our proposed strategy. Intuitively, if the beaconing interval is too long, the roadside infrastructure may miss too many dissemination opportunities. If the beaconing interval is too short, there is no obvious improvement in the energy efficiency. How to obtain a suitable beaconing interval is the most important problem in our study. As shown in Fig. 3, we design a modeling phase and an applying phase in our energy-efficient data dissemination strategy. In the modeling phase, like the traditional data dissemination strategy, the roadside infrastructure sends the beacon packets periodically to learn the presence of passing-by vehicles. The duration of one period defined as $t_{0}$. $t_{0}$ is very small. In the applying phase, the roadside infrastructure calculates the optimal beaconing intervals defined as $\hat{t}_{a}$ and $\hat{t}_{b}$. If the initial beaconing result is 0 , the roadside infrastructure sends the beacon packets with time interval $\hat{t}_{a}$, otherwise with $\hat{t}_{b}$. The detail of the new strategy is shown in the following sections.

\section{The modeling phase}

Normally, the research objects of our paper are deployed on the highway. The existence of the Markov property in the scenario of highway has been validated in [23-25]. Since the beaconing results are directly related to the presences of the passing-by vehicles, we can model the traditional beaconing procedure as a continuous-time Markov process. We define the state space as $X=\left\{x_{1}, x_{2}\right\}$, with $x_{1}=0$ and $x_{2}=1$ indicating that the beaconing results are 0 and 1 . The state transition probability matrix is defined as $P(t)=\left(\begin{array}{ll}P_{00}(t) & P_{01}(t) \\ P_{10}(t) & P_{11}(t)\end{array}\right)$, where $P_{i j}(t)=$ $P\left(R_{s+t}=j \mid R_{s}=i\right), i, j \in\{0,1\}$.

\subsection{The state transition probabilities when the beaconing period is $t_{0}$}

As shown in Fig. 3, we define $M$ as the number of the beaconing periods in the modeling phase. We define $\mathbf{R}=$ $\left\{R_{1}, R_{2}, \cdots, R_{M}\right\}$ with $R_{m} \in X$ for $\forall m \in\{1, \cdots, M\}$ as the beaconing results. After the $M$ beaconing periods, the roadside infrastructure can get the beaconing result samples denoted as $r_{1}, r_{2}, \cdots, r_{M}$. Based on these samples, we can estimate the state transition probabilities of the Markov model. The state transition probability matrix is

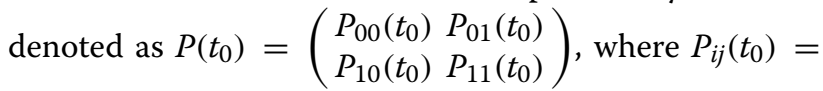
$P\left(R_{s+t_{0}}=j \mid R_{s}=i\right), i, j \in\{0,1\}, s=\left\{1 t_{0}, 2 t_{0}, \cdots,(M-\right.$ 1) $\left.t_{0}\right\}$. Since it can be understood that the discrete-time Markov chain is the discretization of the continuous-time Markov chain, when the time slot is very small, they are identical in essence. According to this property, we use the maximum likelihood estimation method that is used in the discrete-time Markov chain to estimate transition probability. The maximum likelihood estimation is consistent and asymptotically unbiased. The counts of the occurrence of four different transition types such as $\left(r_{k}, r_{k+1}\right)=(0,0),(0,1),(1,0),(1,1)$ are defined as $n_{00}$, $n_{01}, n_{10}$, and $n_{11}, k=\{1,2, \cdots, M-1\}$. The likelihood function is given by

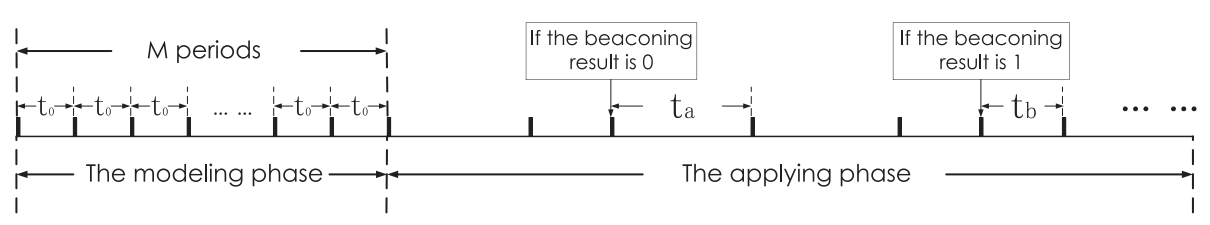

Fig. 3 The beaconing process in our new energy-efficient data dissemination strategy 


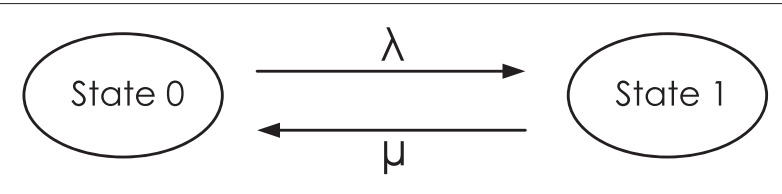

Fig. 4 State transition rate of the Markov process

$$
\begin{aligned}
L\left(P\left(t_{0}\right)\right)= & P\left(\mathbf{R} ; P\left(t_{0}\right)\right) \\
= & P\left(\prod_{k=1}^{M-1} P\left(R_{(k+1) t_{0}}=r_{k+1} \mid R_{k t_{0}}=r_{k} ; A\right)\right. \\
= & P\left(R_{1}=r_{1} ; P\left(t_{0}\right)\right) \cdot\left(P_{00}\left(t_{0}\right)^{n_{00}} \cdot P_{01}\left(t_{0}\right)^{n_{01}}\right. \\
& \left.\cdot P_{10}\left(t_{0}\right)^{n_{10}} \cdot P_{11}\left(t_{0}\right)^{n_{11}}\right) .
\end{aligned}
$$

By solving

$$
\left\{\begin{array}{l}
\partial L\left(P\left(t_{0}\right)\right) / \partial P_{00}\left(t_{0}\right)=0 \\
\partial L\left(P\left(t_{0}\right)\right) / \partial P_{01}\left(t_{0}\right)=0 \\
\partial L\left(P\left(t_{0}\right)\right) / \partial P_{10}\left(t_{0}\right)=0 \\
\partial L\left(P\left(t_{0}\right)\right) / \partial P_{11}\left(t_{0}\right)=0,
\end{array}\right.
$$

the maximum likelihood estimators $\hat{P_{00}}\left(t_{0}\right), \hat{P_{01}}\left(t_{0}\right)$, $\hat{P_{10}}\left(t_{0}\right)$, and $\hat{P_{11}}\left(t_{0}\right)$ can be obtained as follows:

$$
\left\{\begin{array}{l}
\hat{P_{00}}\left(t_{0}\right)=n_{00} /\left(n_{00}+n_{01}\right) \\
\hat{P_{01}}\left(t_{0}\right)=n_{01} /\left(n_{00}+n_{01}\right) \\
\hat{P_{10}}\left(t_{0}\right)=n_{10} /\left(n_{10}+n_{11}\right) \\
\hat{P_{11}}\left(t_{0}\right)=n_{11} /\left(n_{10}+n_{11}\right) .
\end{array}\right.
$$

Then, the estimation process of the state transition probabilities has been done.

\subsection{The duration of the modeling phase}

As described above, if we have the samples, we can make the estimation. It is obvious that the more samples we get, the more accurate the estimation is. An analysis of how many samples are needed to satisfy the desired accuracy is presented in this subsection.

The central limit theorem is utilized in our analysis.

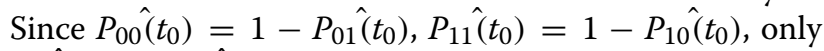
$P_{01}\left(t_{0}\right)$ and $P_{10}\left(t_{0}\right)$ will be discussed in this subsection. We discuss $\hat{P_{01}}\left(t_{0}\right)$ first. Assuming among $M$ samples there are $M_{0}$ ones whose values are 0 , then we have

$$
M_{0}=M \cdot\left(1-\frac{P_{01}\left(t_{0}\right)}{P_{01}\left(t_{0}\right)+P_{10}\left(t_{0}\right)}\right) \text { as } M \rightarrow \infty
$$

where $\frac{P_{01}\left(t_{0}\right)}{P_{01}\left(t_{0}\right)+P_{10}\left(t_{0}\right)}$ represents the probability that the beaconing result is 1 . We define

$$
C_{k}=\left\{\begin{array}{ll}
0 & r_{k}=0, r_{k+1}=0 \\
1 & r_{k}=0, r_{k+1}=1
\end{array},\left(k=1, \cdots, M_{0}\right) .\right.
$$

$\left\{C_{k}\right\}$ are independent and identically distributed variables. The mean value of $\left\{C_{k}\right\}$ is $P_{01}\left(t_{0}\right)$, and the variance is $P_{01}\left(t_{0}\right)-P_{01}\left(t_{0}\right)^{2}$. From (3), $\hat{P_{01}}\left(t_{0}\right)$ can be expressed as

$$
\hat{P_{01}}\left(t_{0}\right)=n_{01} /\left(n_{00}+n_{01}\right)=\frac{1}{M_{0}} \sum_{k=1}^{M_{0}} C_{k} .
$$

We demand that the relative error $\left|\hat{P_{01}}\left(t_{0}\right)-P_{01}\left(t_{0}\right)\right|$ of an estimator should be below a relative estimation error $\theta$ with the corresponding confidence probability $\alpha$. In mathematical terms, we request

$$
P\left(\left|\frac{\hat{P_{01}}\left(t_{0}\right)-P_{01}\left(t_{0}\right)}{P_{01}\left(t_{0}\right)}\right|<\theta\right) \geq \alpha,
$$

where $\theta$ and $\alpha$ are inputs to describe the desired accuracy in the estimation. If (7) is fulfilled, the estimation achieves the desired accuracy. Combining (4) and (7), since $P_{01}\left(t_{0}\right) \geq 0$, we have following equations:

$$
\begin{aligned}
& P\left(\left|\frac{P_{01}\left(t_{0}\right)-P_{01}\left(t_{0}\right)}{P_{01}\left(t_{0}\right)}\right|<\theta\right) \\
= & P\left(\left|\frac{1}{M_{0}} \sum_{k=1}^{M_{0}} C_{k}-P_{01}\left(t_{0}\right)\right|<\theta P_{01}\left(t_{0}\right)\right) \\
= & P\left(\left|\frac{\sum_{k=1}^{M_{0}} C_{k}-M_{0} P_{01}\left(t_{0}\right)}{\sqrt{M_{0}} \cdot \sqrt{P_{01}\left(t_{0}\right)-P_{01}\left(t_{0}\right)^{2}}} \cdot \frac{\sqrt{P_{01}\left(t_{0}\right)-P_{01}\left(t_{0}\right)^{2}}}{\sqrt{M_{0}}}\right|<\theta P_{01}\left(t_{0}\right)\right) \\
= & P\left(\left|\frac{\sum_{k=1}^{M_{0}} C_{k}-M_{0} P_{01}\left(t_{0}\right)}{\sqrt{M_{0}} \cdot \sqrt{P_{01}\left(t_{0}\right)-P_{01}\left(t_{0}\right)^{2}}}\right|<\theta \frac{\sqrt{M_{0}}}{\sqrt{\frac{1}{P_{01}\left(t_{0}\right)}-1}}\right) \\
= & P\left(\left|\frac{\sum_{k=1}^{M_{0}} C_{k}-M_{0} P_{01}\left(t_{0}\right)}{\sqrt{M_{0}} \cdot \sqrt{P_{01}\left(t_{0}\right)-P_{01}\left(t_{0}\right)^{2}}}\right|<\theta \frac{\sqrt{M \cdot\left(1-\frac{P_{01}\left(t_{0}\right)}{P_{01}\left(t_{0}\right)+P_{10}\left(t_{0}\right)}\right)}}{\sqrt{\frac{1}{P_{01}\left(t_{0}\right)}-1}}\right) .
\end{aligned}
$$

Based on the central limit theorem, we have

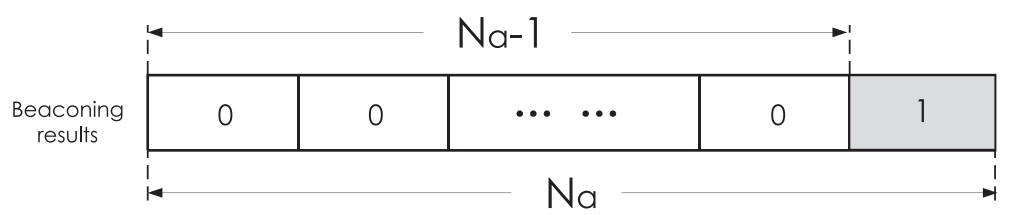

Fig. 5 The case when the initial beaconing result is 0 
Table 2 The relationship between $E T_{a}\left(t_{a}\right)$ and $t_{a}$

\begin{tabular}{lcccccc}
\hline$E T_{a}\left(t_{a}\right)$ & 0.5 & 1.0 & 1.5 & 2.0 & 2.5 & 3.0 \\
$t_{a}$ & 0.28 & 0.59 & 0.91 & 1.23 & 1.49 & 1.82 \\
\hline
\end{tabular}

$$
\frac{\sum_{k=1}^{M_{0}} C_{k}-M_{0} P_{01}\left(t_{0}\right)}{\sqrt{M_{0}} \cdot \sqrt{P_{01}\left(t_{0}\right)-P_{01}\left(t_{0}\right)^{2}}} \rightarrow N(0,1) \text { as } M_{0} \rightarrow \infty .
$$

We denote $\Phi(\bullet)$ as the standard normal cumulative distribution function. Then, we have

$$
2 \Phi\left(\theta \frac{\sqrt{M \cdot\left(1-\frac{P_{01}\left(t_{0}\right)}{P_{01}\left(t_{0}\right)+P_{10}\left(t_{0}\right)}\right)}}{\sqrt{\frac{1}{P_{01}\left(t_{0}\right)}-1}}\right)-1 \geq \alpha .
$$

In (10), $M>0,0 \leq P_{01}\left(t_{0}\right) \leq 1$ and $0 \leq P_{10}\left(t_{0}\right) \leq 1$. We denote $M_{\min }^{a}$ as the minimum required number of the beaconing result samples for $\hat{P_{01}}\left(t_{0}\right)$. For any state transition probability values, $M^{a}$ can be expressed as follows:

$M_{\min }^{a}=\frac{\left(\Phi^{-1}\left(\frac{1+\alpha}{2}\right)\right)^{2}}{\theta^{2}}\left(1-P_{01}\left(t_{0}\right)\right)\left(\frac{1}{P_{01}\left(t_{0}\right)}+\frac{1}{P_{10}\left(t_{0}\right)}\right)$.

For $\hat{P_{10}}\left(t_{0}\right)$, the analysis process is the same. The minimum required number of the beaconing result samples for $\hat{P_{01}}\left(t_{0}\right)$ is denoted as $M_{\min }^{b}$. We have

$M_{\min }^{b}=\frac{\left(\Phi^{-1}\left(\frac{1+\alpha}{2}\right)\right)^{2}}{\theta^{2}}\left(1-P_{10}\left(t_{0}\right)\right)\left(\frac{1}{P_{01}\left(t_{0}\right)}+\frac{1}{P_{10}\left(t_{0}\right)}\right)$

Therefore, when the desired accuracy $\alpha$ and $\theta$ are given, we have the following equations:

$$
M_{\min }=\max \left\{M_{\min }^{a}, M_{\min }^{b}\right\},
$$

where $M_{\min }$ is denoted as the minimum required number of the beaconing result samples for the estimation.

Actually, since we do not know the values of $P_{01}\left(t_{0}\right)$ and $P_{10}\left(t_{0}\right)$ before we start the modeling phase, we cannot calculate the required number of the samples. To tackle this problem, we have the following processes. At the beginning of the modeling phase, we send $M_{\text {default }}$ beacon packets firstly. The value of $M_{\text {default }}$ is analyzed in the later section. According to the $M_{\text {default }}$ beaconing result samples, we can calculate the $P_{01}\left(t_{0}\right)$ and $P_{10}\left(t_{0}\right)$. Then, we can get the $M_{\min }$. If $M_{\min } \leq M_{\text {default }}$, it means the estimation of the state transition probabilities reaches the desired accuracy, and we can stop the modeling phase. If not, the periodical beaconing continues. We denote $M_{\text {actual }}$ as the actual number of the beacon packets. After each of the continuous beaconing processes, we recalculate the $M_{\min }$ and compare it with the $M_{\text {actual }}$. Until $M_{\text {actual }} \geq M_{\min }$, we stop the periodical beaconing and start the applying phase. The total beaconing times of the modeling phase $M_{\text {total }}$ is equal to the current $M_{\text {actual }}$. The total duration of the modeling phase is expressed as

$$
T_{\text {modeling }}=t_{0} M_{\text {total }}
$$

Through the above analyses, we can summarize the following proposition:

Proposition 1. After acquiring a certain number of samples, the estimation of the state transition probabilities for the Markov process can achieve a desired accuracy.

\section{The applying phase}

After obtaining the state transition probabilities when the beaconing period is $t_{0}$, we can calculate the system parameters of the Markov process. Then, we can obtain the optimal beaconing interval and apply it in the applying phase.

\subsection{The state transition probabilities in the applying phase}

We define the state transition rate matrix of the Markov process as

$$
Q=\left(\begin{array}{cc}
-\lambda & \lambda \\
\mu & -\mu
\end{array}\right)
$$

where $\lambda$ and $\mu$ are the arrival rate and the departure rate shown in Fig. 4. The state transition probabilities can be derived from the matrix $\mathrm{Q}$. We have
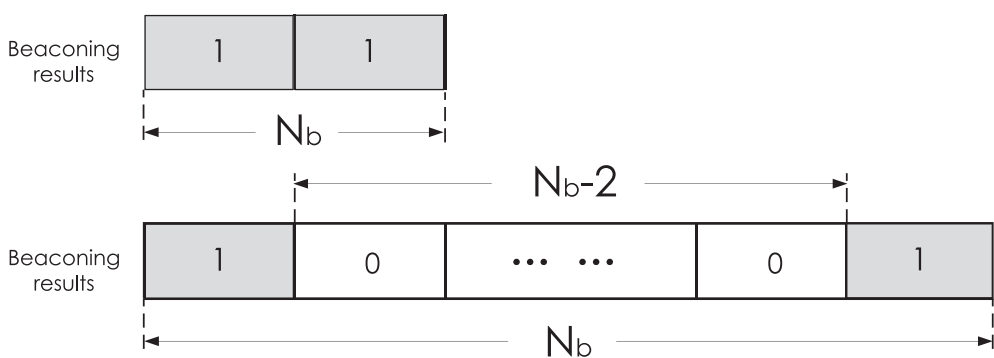

Fig. 6 The case when the initial beaconing result is 1 
Table 3 The relationship between $E T_{a}\left(t_{b}\right), t_{a}$, and $t_{b}$

\begin{tabular}{lcccccc}
\hline$E T_{b}\left(t_{b}\right)$ & 0.5 & 1.0 & 1.5 & 2.0 & 2.5 & 3.0 \\
\hline$t_{a}$ & 0.28 & 0.59 & 0.91 & 1.23 & 1.49 & 1.82 \\
$t_{b}$ & 0.23 & 0.49 & 0.78 & 1.11 & 1.30 & 1.57 \\
\hline
\end{tabular}

$$
\left\{\begin{array}{l}
P_{00}(t)=\frac{\mu}{\lambda+\mu}+\frac{\lambda}{\lambda+\mu} e^{-(\lambda+\mu) t} \\
P_{01}(t)=\frac{\lambda}{\lambda+\mu}-\frac{\lambda}{\lambda+\mu} e^{-(\lambda+\mu) t} \\
P_{10}(t)=\frac{\mu}{\lambda+\mu}-\frac{\mu}{\lambda+\mu} e^{-(\lambda+\mu) t} \\
P_{11}(t)=\frac{\lambda}{\lambda+\mu}+\frac{\mu}{\lambda+\mu} e^{-(\lambda+\mu) t}
\end{array}\right.
$$

In the modeling phase, we have obtained the estimation results $\hat{P_{00}}\left(t_{0}\right), \hat{P_{01}}\left(t_{0}\right), \hat{P_{10}}\left(t_{0}\right)$, and $\hat{P_{11}}\left(t_{0}\right)$. Combining with the beaconing period $t_{0}$, we obtain

$$
\left\{\begin{array}{l}
\lambda=\frac{\left(\hat{P_{01}}\left(t_{0}\right)\right) \log \left(\frac{1}{1-\hat{P}_{01}\left(t_{0}\right)-P_{10}\left(t_{0}\right)}\right)}{\left(\hat{P_{01}}\left(t_{0}\right)+\hat{P_{10}}\left(t_{0}\right)\right) t_{0}} \\
\mu=\frac{\left(\hat{P_{10}}\left(t_{0}\right)\right) \log \left(\frac{1}{1-P_{01}\left(t_{0}\right)-P_{10}\left(t_{0}\right)}\right)}{\left(\hat{P_{01}}\left(t_{0}\right)+\hat{P_{10}}\left(t_{0}\right)\right) t_{0}}
\end{array} .\right.
$$

According to (16) and (17), we can calculate the state transition probabilities in any value of $t$.

\subsection{The optimal beaconing interval when the initial beaconing result is 0}

Figure 5 illustrates the case when the initial beaconing result is 0 . We denote $N_{a}$ as the beaconing times such that the roadside infrastructure can discover one passing-by vehicle in this case. Obviously, the first $N_{a}-1$ beaconing results are 0 , the final one is 1 . The probability mass function of $N_{a}$ can be represented as

$$
P\left(N_{a}=n\right)=\left\{\begin{array}{ll}
P_{01}\left(t_{a}\right) P_{00}^{n-1}\left(t_{a}\right) & n \geq 1 \\
0 & \text { others }
\end{array} .\right.
$$

Table 4 Simulation parameters

\begin{tabular}{ll}
\hline Parameter & Value \\
\hline The length of a beaconing period to & $0.1 \mathrm{~s}$ \\
The confidence probability value $\alpha$ & $99 \%$ \\
Relative estimation error $\theta$ & $20 \%$ \\
Energy used for each beaconing $\xi$ & $10 \mathrm{~mJ}$ \\
Average amount of data in each transmission process $\omega$ & $300 \mathrm{kbit}$ \\
Threshold $\delta_{E}^{a}$ & 90 \\
Threshold $\delta_{R}^{a}$ & 5.5 \\
Threshold $\delta_{E}^{b}$ & 16 \\
Threshold $\delta_{R}^{b}$ & 600 \\
\hline
\end{tabular}

The expectation of the required beaconing times $E N_{a}\left(t_{a}\right)$ is

$$
\begin{aligned}
E N_{a}\left(t_{a}\right) & =\sum_{n=1}^{\infty} n P_{01}\left(t_{a}\right) P_{00}^{n-1}\left(t_{a}\right) \\
& =\frac{P_{01}\left(t_{a}\right)}{1+P_{00}^{2}\left(t_{a}\right)-2 P_{00}\left(t_{a}\right)} .
\end{aligned}
$$

Therefore, the expectation of single-vehicle discovery time $E T_{a}\left(t_{a}\right)$ is

$$
E T_{a}\left(t_{a}\right)=t_{a} \cdot E N_{a}\left(t_{a}\right)
$$

When $\hat{P_{01}}\left(t_{0}\right)=0.28$ and $\hat{P_{10}}\left(t_{0}\right)=0.40$, we have Table 2.

All the calculated beaconing intervals are larger than $t_{0}=0.1$. The energy consumption decreases.

\subsubsection{The maximum beaconing interval}

We denote the average data transmission rate requirement as $D$. The average amount of data in each of data transmission process is denoted as $\omega$. Then, we have

$$
D \leq \frac{\omega}{E T_{a}\left(t_{a}\right)}
$$

According to (18), (19), and (20), we can calculate the maximum $t_{a}$ which is denoted as $t_{a}^{\max }$.

\subsubsection{The optimal beaconing interval}

Generally, in the real system, we do not always need to use the maximum beaconing interval. Under the condition that there is an average data transmission rate requirement, we need to calculate an optimal beaconing interval that can balance the tradeoff between energy consumption and the average data transmission rate. In this paper, we introduce a satisfaction degree based on the Sigmoid function to combine the decreased energy

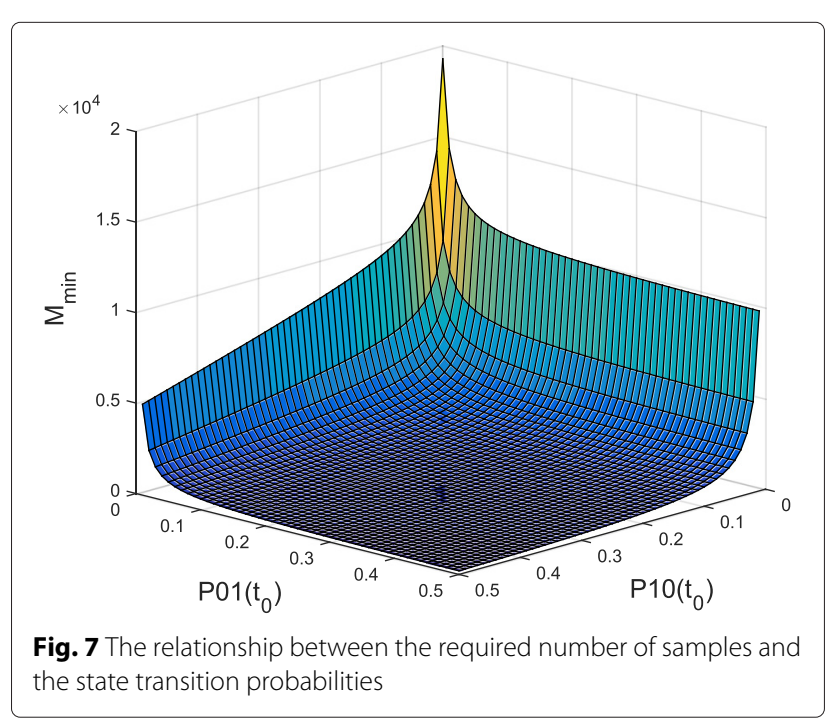


consumption and the decreased data rate effectively. The Sigmoid function has been widely employed to approximate users' satisfaction with respect to service quality or resource allocation. The optimal beaconing interval calculation process is shown as follows.

When the initial beaconing result is 0 , the average decreased energy consumption is denoted as $f_{E}\left(t_{a}\right)$, the average decreased data rate is denoted as $f_{R}\left(t_{a}\right)$. The energy used for each beaconing is denoted as $\xi$. Then, we have

$$
\begin{gathered}
f_{E}\left(t_{a}\right)=\xi\left(\frac{1}{t_{0}}-\frac{1}{t_{a}}\right) \\
f_{R}\left(t_{a}\right)=\omega\left(\frac{1}{E T_{a}\left(t_{0}\right)}-\frac{1}{E T_{a}\left(t_{a}\right)}\right) .
\end{gathered}
$$

We normalize $f_{E}\left(t_{a}\right)$ and $f_{R}\left(t_{a}\right)$ to the range $[0,1]$ to linearly combine them. We denote $\widehat{f}_{E}\left(t_{a}\right)$ as a measure of the degree of satisfaction for the decreased energy consumption. We denote $\widehat{f_{R}}\left(t_{a}\right)$ as a measure of the degree of satisfaction for the decreased data rate. The normalization process is shown as follows:

$$
\begin{gathered}
\widehat{f_{E}}\left(t_{a}\right)=\frac{1}{1+\exp \left(-\left(f_{E}\left(t_{a}\right)-\delta_{E}^{a}\right)\right)} \\
\widehat{f_{R}}\left(t_{a}\right)=-\frac{1}{1+\exp \left(-\left(f_{R}\left(t_{a}\right)-\delta_{R}^{a}\right)\right)} .
\end{gathered}
$$

In the above, $\widehat{f_{E}}\left(t_{a}\right)$ is modeled as a Sigmoid function of $f_{E}\left(t_{a}\right) . \delta_{E}^{a}$ is a predefined threshold reflecting

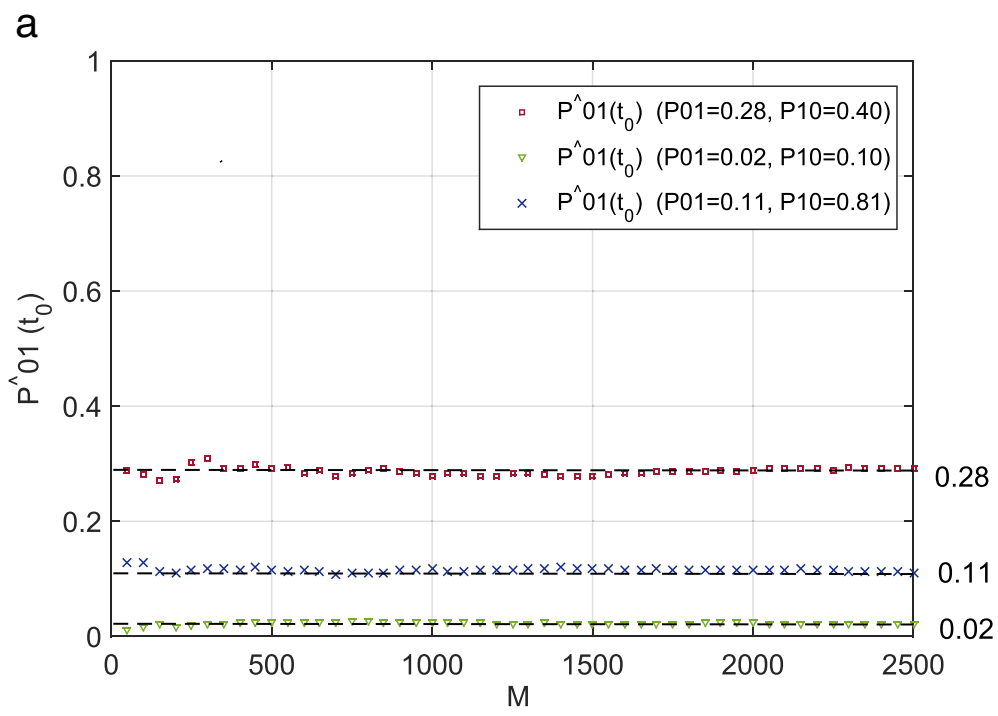

b

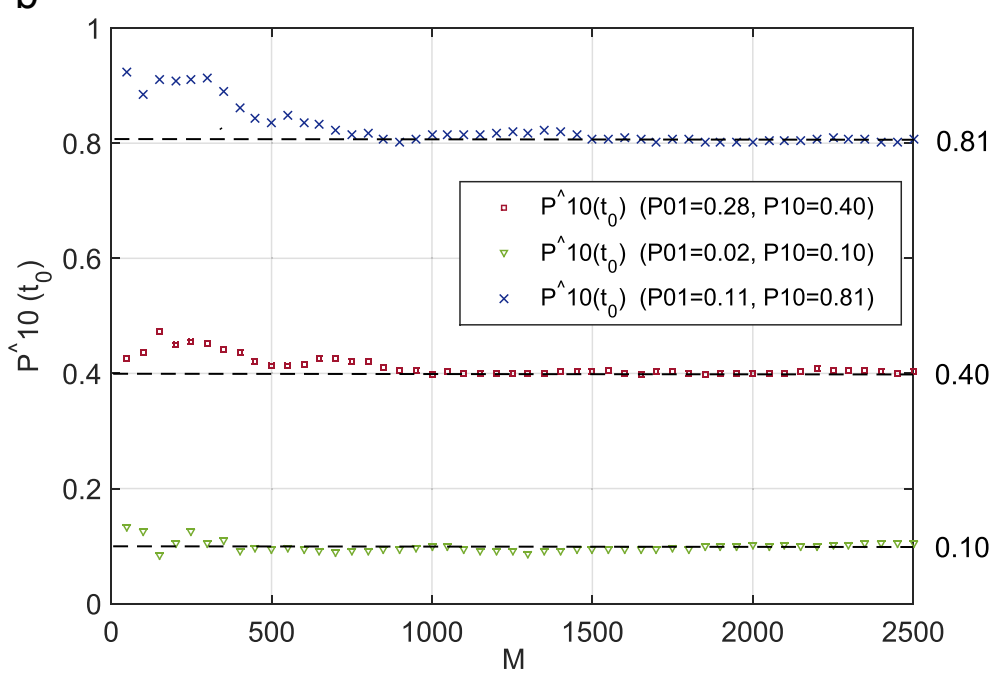

Fig. 8 The relationship between the number of samples and the value of maximum likelihood estimation. a Effects of $\xi$ on $g_{x}$. a Consider $P_{01}\left(t_{0}\right)$. $\mathbf{b}$ Consider $P_{10}\left(t_{0}\right)$ 


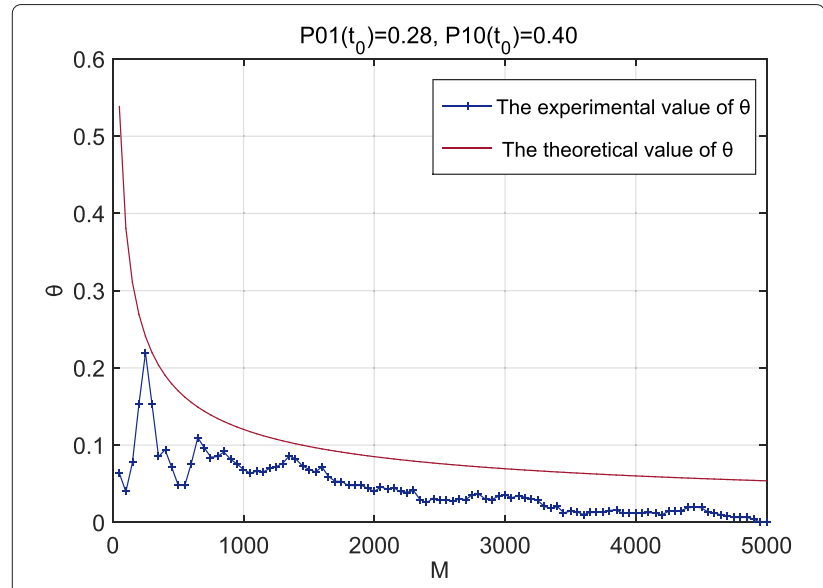

Fig. 9 The relative estimation error of the maximum likelihood estimation

the requirement of the decreased energy consumption, below which the roadside infrastructure has very limited satisfaction and above which its satisfaction rapidly reaches an asymptotic value. $\delta_{R}^{a}$ is defined similarly. In this paper, a roadside infrastructure can define the thresholds $\delta_{E}^{a}$ and $\delta_{R}^{a}$ according to its own requirements. The effects of $\delta_{E}^{a}$ and $\delta_{R}^{a}$ are analyzed in the later section.

It should be noticed that $\widehat{f_{E}}\left(t_{a}\right)$ is a positive valuedecreasing function since the degree of satisfaction decreases with the increase of the energy consumption. On the other hand, $\widehat{f_{R}}\left(t_{a}\right)$ is a positive value-increasing function, which implies that higher single-vehicle discovery rate leads to more satisfaction of the roadside infrastructure.

By jointly considering $\widehat{f_{E}}\left(t_{a}\right)$ and $\widehat{f_{R}}\left(t_{a}\right)$, the objective function $f_{X}$ is defined as

$$
f_{X}=\widehat{f_{E}}\left(t_{a}\right)+\widehat{f_{R}}\left(t_{a}\right)
$$

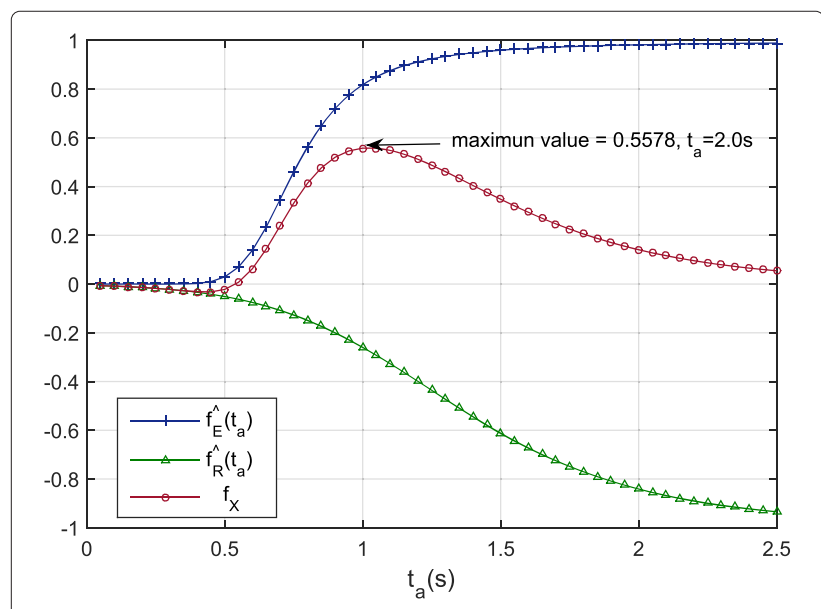

Fig. 10 The optimal interval when the initial beaconing result is 0
When the initial beaconing result is 0 , by maximizing (26) and considering $t_{a}^{\max }$, we can obtain the optimal beaconing interval $\hat{t}_{a}$

$$
\hat{t}_{a}=\min \left\{t_{a}^{\max }, \arg \max f_{X}\right\} .
$$

\subsection{The optimal beaconing interval when the initial beaconing result is 1}

Figure 6 illustrates the case when the initial beaconing result is 1 . We denote $N_{b}$ as the beaconing times such that the roadside infrastructure can discover one passing-by vehicle in this case. Obviously, except the first and the final beaconing results, the left results are 0 . Considering that the probability of $N_{b}=1$, denoted as $P\left(N_{b}=1\right)$, is different from other probabilities, the probability mass function of $N_{b}$ can be represented as

$$
P\left(N_{b}=n\right)=\left\{\begin{array}{ll}
P_{11}\left(t_{b}\right) & n=1 \\
P_{10}\left(t_{b}\right) P_{01}\left(\hat{t}_{a}\right) P_{00}^{n-2}\left(\hat{t}_{a}\right) & n \geq 2 \\
0 & \text { others }
\end{array},\right.
$$

where $\hat{t}_{a}$ is the optimal beaconing interval obtained in the above subsection. The expectation of required beaconing times $E N_{b}\left(t_{b}\right)$ is

$$
\begin{aligned}
E N_{b}\left(t_{b}\right) & =P_{11}\left(t_{b}\right)+\sum_{n=2}^{\infty} n P_{10}\left(t_{b}\right) P_{01}\left(t_{a}\right) P_{00}^{n-2}\left(t_{a}\right) \\
& =P_{11}\left(t_{b}\right)+\left(\frac{P_{10}\left(t_{b}\right) P_{01}\left(t_{a}\right)\left(2-P_{00}\left(t_{a}\right)\right)}{1+P_{00}^{2}\left(t_{a}\right)-2 P_{00}\left(t_{a}\right)}\right) .
\end{aligned}
$$

Therefore, the expectation of single-vehicle discovery time $E T_{b}\left(t_{b}\right)$ is

$$
\begin{aligned}
E T_{b}\left(t_{b}\right)= & t_{b} P_{11}\left(t_{b}\right)+\sum_{n=2}^{\infty}\left(t_{b}+(n-1) t_{a}\right) P_{10}\left(t_{b}\right) P_{01}\left(t_{a}\right) P_{00}^{n-2}\left(t_{a}\right) \\
= & t_{b} P_{11}\left(t_{b}\right) \\
& +\frac{P_{10}\left(t_{b}\right) P_{01}\left(t_{a}\right)\left(\left(t_{b}-t_{a}\right)\left(1-P_{00}\left(t_{a}\right)\right)-P_{00}\left(t_{a}\right)+2\right)}{1+P_{00}^{2}\left(t_{a}\right)-2 P_{00}\left(t_{a}\right)} .
\end{aligned}
$$

When $\hat{P_{01}}\left(t_{0}\right)=0.28$ and $\hat{P_{10}}\left(t_{0}\right)=0.40$, we have Table 3.

All the calculated beaconing intervals are larger than $t_{0}=0.1$. The energy consumption decreases.

\subsubsection{The maximum beaconing interval}

We denote the average data transmission rate requirement as $D$. The average amount of data in each of data transmission process is denoted as $\omega$. Then, we have

$$
D \leq \frac{\omega}{E T_{b}\left(t_{b}\right)} .
$$


According to (18), (19), and (20), we can calculate the maximum $t_{b}$ which is denoted as $t_{b}^{\max }$.

\subsubsection{The optimal beaconing interval}

Similarly, based on the Sigmoid function, we can obtain the optimal beaconing interval as follows. We denote the average decreased energy consumption as $g_{E}\left(t_{b}\right)$. The average decreased data rate is denoted as $g_{R}\left(t_{b}\right)$. Then, we have

$$
\begin{aligned}
& g_{E}\left(t_{b}\right)=\xi\left(\frac{E N_{b}\left(t_{0}\right)}{E T_{b}\left(t_{0}\right)}-\frac{E N_{b}\left(t_{b}\right)}{E T_{b}\left(t_{b}\right)}\right) \\
& g_{R}\left(t_{b}\right)=\omega\left(\frac{1}{E T_{b}\left(t_{0}\right)}-\frac{1}{E T_{b}\left(t_{b}\right)}\right) .
\end{aligned}
$$

When the initial beaconing result is 1 , we denote $\widehat{g_{E}}\left(t_{b}\right)$ as the measure of the degree of satisfaction for the decreased energy consumption, and we denote $\widehat{g_{E}}\left(t_{b}\right)$ as the Sigmoid function of $f_{E}\left(t_{a}\right) . \delta_{E}^{b}$ is a predefined threshold reflecting the energy requirement, and $\delta_{R}^{b}$ is a predefined threshold reflecting the data rate requirement. We denote $g_{X}$ as the objective function. Similar with the optimization process in the above subsection, we have

$$
\begin{gathered}
\widehat{g_{E}}\left(t_{b}\right)=\frac{1}{1+\exp \left(-\left(g_{E}\left(t_{b}\right)-\delta_{E}^{b}\right)\right)} \\
\widehat{g_{R}}\left(t_{b}\right)=-\frac{1}{1+\exp \left(-\left(g_{R}\left(t_{b}\right)-\delta_{R}^{b}\right)\right)} \\
g_{X}=\widehat{g_{E}}\left(t_{b}\right)+\widehat{g_{R}}\left(t_{b}\right) .
\end{gathered}
$$

Then, we can obtain the optimal beaconing interval as $\hat{t}_{b}$

$$
\hat{t}_{b}=\min \left\{t_{b}^{\max }, \arg \max g_{X}\right\} .
$$

Through the above analyses, we can summarize the following proposition:

Proposition 2. No matter the initial beaconing result is 0 or 1, the optimal beaconing interval for the roadside infrastructure can be obtained by exploiting the tradeoff between the average energy consumption and the singlevehicle discovery rate.

\subsection{Restarting the modeling process}

Actually, the state transition probabilities are not constants. When they vary, the beaconing intervals previously calculated are no longer effective in the model. Sometimes, keep using such intervals could lead to a sharp decrease on single-vehicle discover rate. Thus, we need to restart the modeling phase to update the beaconing intervals after a certain period. Generally, there are two modes in real application, the first mode is to restart the modeling phase perodically. This mode is easy to implement by presetting a remodeling clock. The other mode is to restart the modeling phase on demand. We compare the expected single-vehicle discovery rate with several recent observed rates. If the difference is beyond a threshold and lasts for a particular length of time, we restart the modeling phase. This mode can better adapt to variability in the model but also holds a higher computational complexity. In this paper, we do not specify which mode we apply. Research on mode selection and parameter determination

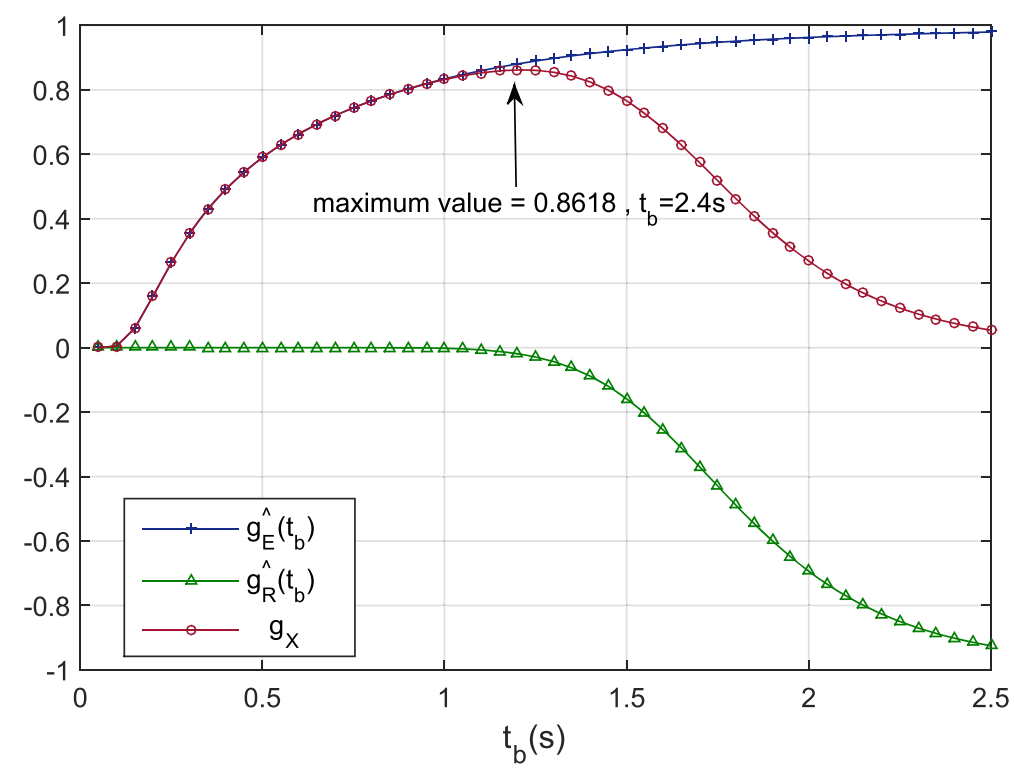

Fig. 11 The optimal interval when the initial beaconing result is 1 
is not our focus in this paper. We leave it to the future work.

\section{Optimal beaconing interval in the data dissemination strategy}

In this paper, we design an energy-efficient data dissemination strategy for roadside infrastructures by optimizing the beaconing interval. The details of the two phase of the new data dissemination strategy are analyzed in the above sections. In this section, we summarize the whole beaconing interval optimization process in Algorithm 1.

\section{Numerical analysis}

The numerical results of the energy-efficient data dissemination strategy are presented in this section. Firstly, we study the performance of the maximum likelihood estimation of the state transition probabilities. Secondly, a specific case is given to show how to choose the optimal

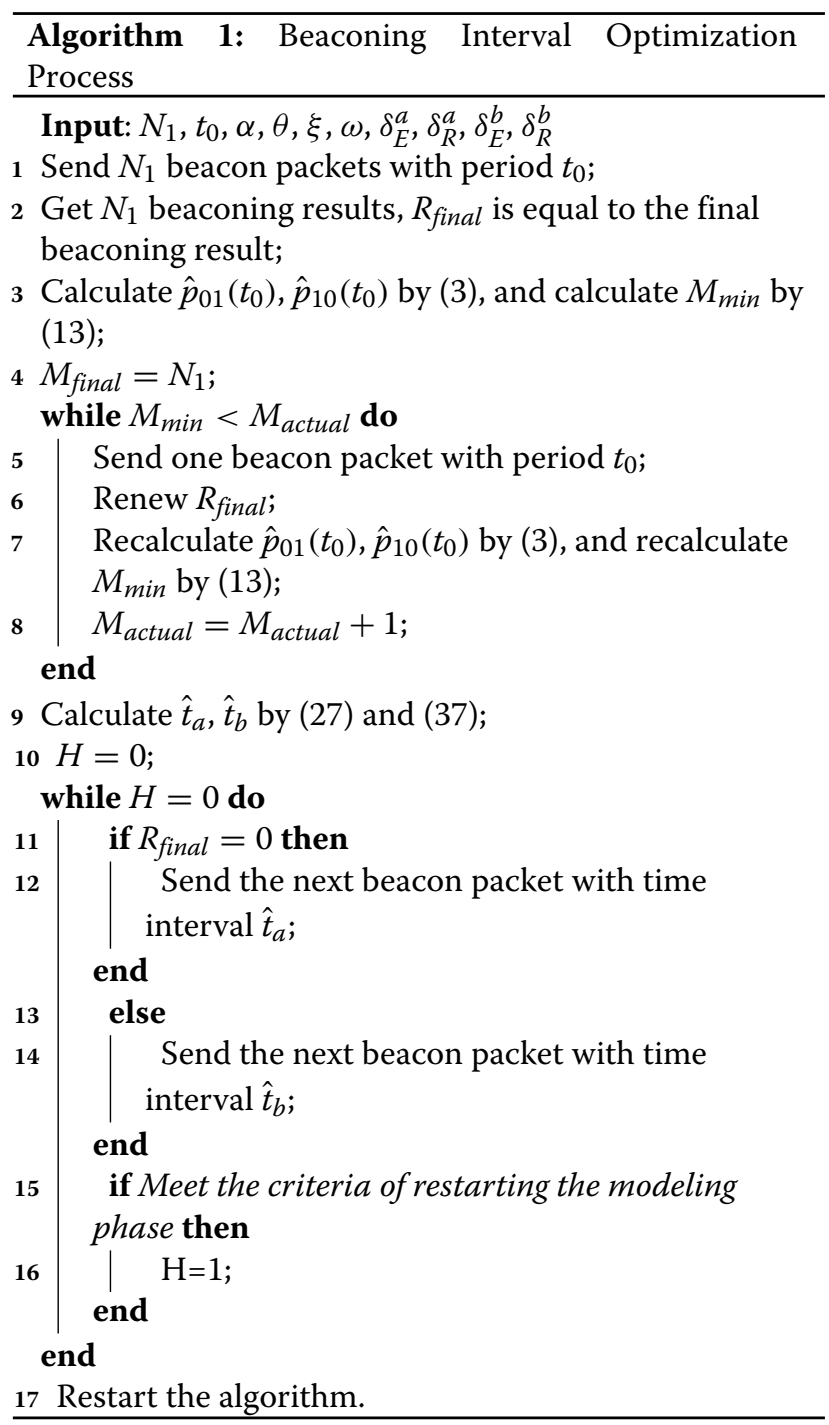

intervals by maximizing the objective functions. Finally, we extensively investigate the effects of the system parameters (denoted by $\xi, \omega, \delta_{E}^{a}, \delta_{R}^{a}, \delta_{E}^{b}, \delta_{R}^{b}$ ) on the optimal beaconing intervals.

\subsection{The performance of the maximum likelihood estimation}

In this subsection, we investigate the accuracy of the maximum likelihood estimation. In Fig. 7, we show the relationship between the required number of samples and the state transition probabilities $\left(P_{01}\left(t_{0}\right), P_{10}\left(t_{0}\right)\right)$ with the given relative estimation error $\theta$ and the confidence probability value $\alpha$ as Table 4 . From this figure, we observe that when $P_{01}\left(t_{0}\right)$ or $P_{10}\left(t_{0}\right)$ decreases, the required number of samples increases.

In Fig. 8a, b, we study the relationship between the number of samples and the value of maximum likelihood estimation. Each point in the figures indicates the value of an estimation. We mark the actual state transition probabilities on the figures. In the figures, we can find that, in the cases of $\left(P_{01}\left(t_{0}\right), P_{10}\left(t_{0}\right)\right)=$ $(0.28,0.40),(0.02,0.10),(0.11,0.81)$, the estimates can approach to the actual state transition probabilities with the number of samples increased.

Figure 9 shows the relative estimation error of the maximum likelihood estimation. The theoretical maximum relative estimation error and the actual relative estimation error are given in this figure. Similar with the results of Fig. $8 \mathrm{a}, \mathrm{b}$, we can find that the relative estimation error decreases with the increase of the number of samples. On the other hand, it is notable that the actual relative estimation error is always smaller than the theoretical value. This proves the validity of our method if calculating the minimum required number of samples.

\subsection{A specific case}

With the parameters in Table $4, \hat{P_{01}}\left(t_{0}\right)=0.28$ and $\hat{P_{10}}\left(t_{0}\right)=0.40$, a specific case is given to show how to choose the optimal intervals by maximizing the objective functions. Figure 10 corresponds to that the initial beaconing result is 0 . In Fig. 10, we observe that $\widehat{f_{E}}\left(t_{a}\right)$ increases with $t_{a}$ while $\widehat{f_{R}}\left(t_{a}\right)$ decreases with $t_{a}$. The reason is that with the increase of the beaconing interval $t_{a}$, the average decreased energy gets larger and also the decreased data rate becomes larger, thus leading to a higher satisfaction on energy consumption and a higher dissatisfaction on data rate. By carefully balancing the decreased energy consumption and the decreased data rate, the optimal beaconing interval can be found. Figure 11 corresponds to that the initial beaconing result is 1 . The increase of $\widehat{g_{E}}\left(t_{b}\right)$ and the decrease of $\widehat{g_{R}}\left(t_{b}\right)$ can be explained similarly as in Fig. 10. Another important observation is that when the initial beaconing results are different, the optimal values are different. Particularly, 


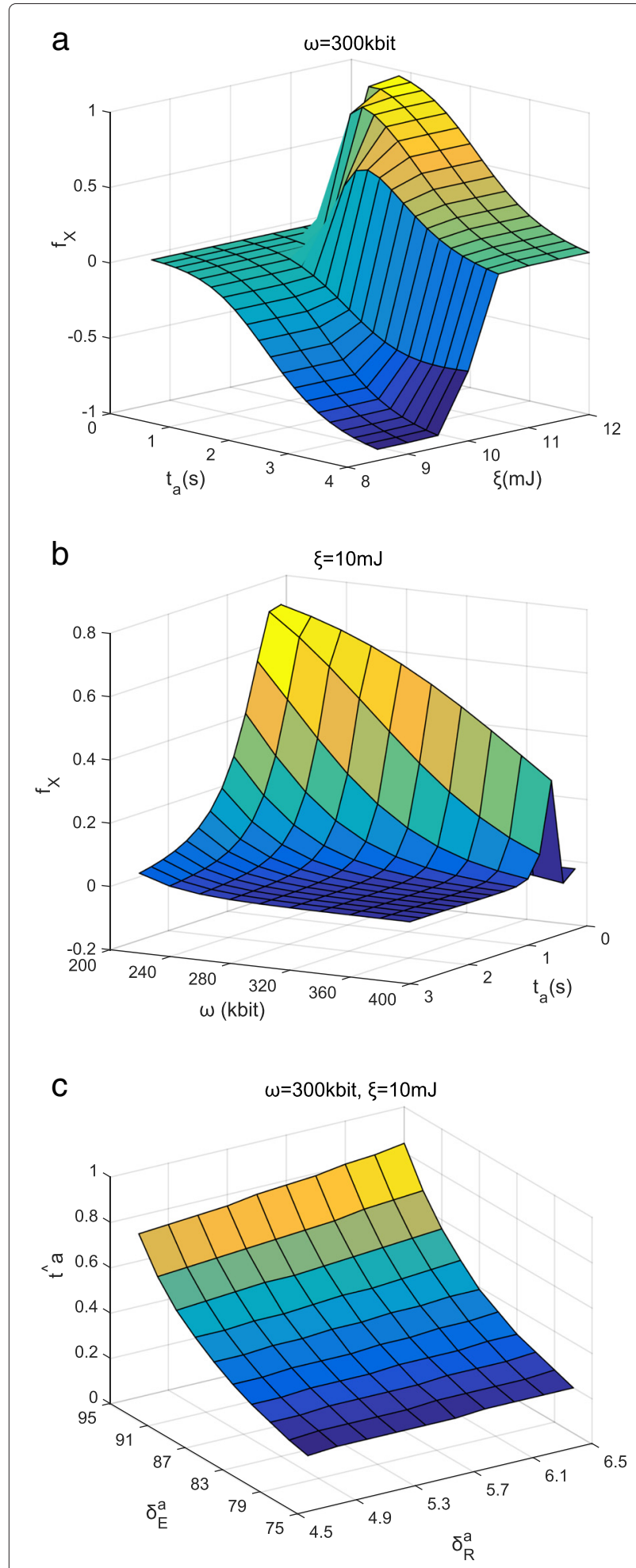

Fig. 12 Effect of the system parameters when initially the state is $0 . \mathbf{a}$ Effects of $\xi$ on $f_{X} \cdot \mathbf{b}$ Effects of $\omega$ on $f_{X}$. c Effects of $\delta_{E}^{a}, \delta_{R}^{a}$ on $\hat{t}_{a}$

when the maximum value of $f_{X}$ is 0.5578 , the optimal beaconing interval $\hat{t}_{a}$ is $2 \mathrm{~s}$; while when the maximum value of $g_{X}$ is 0.8618 , the optimal beaconing interval $\hat{t}_{b}$ is $2.4 \mathrm{~s}$.

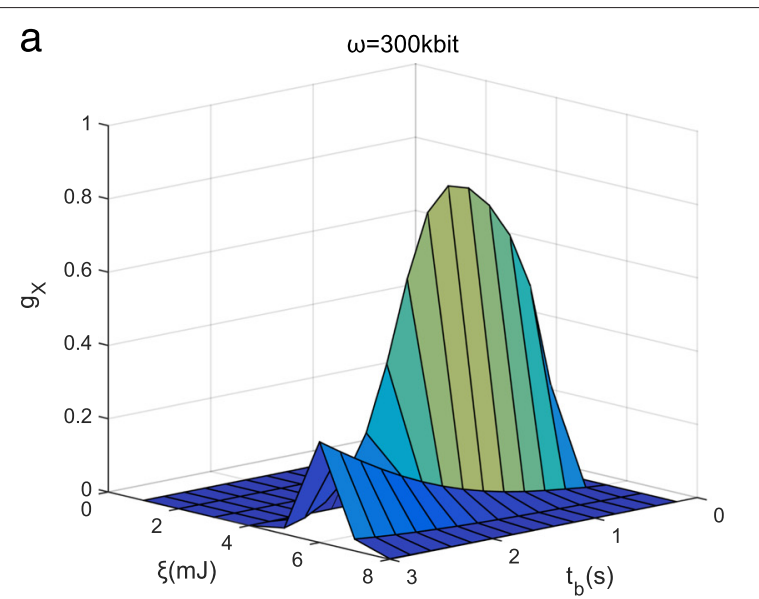

b

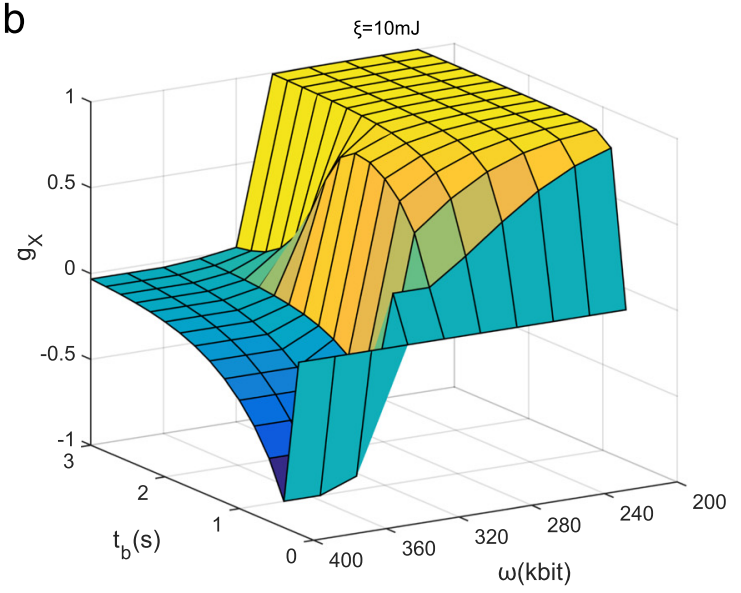

C $\quad \omega=300 \mathrm{kbit}, \xi=10 \mathrm{~mJ}$

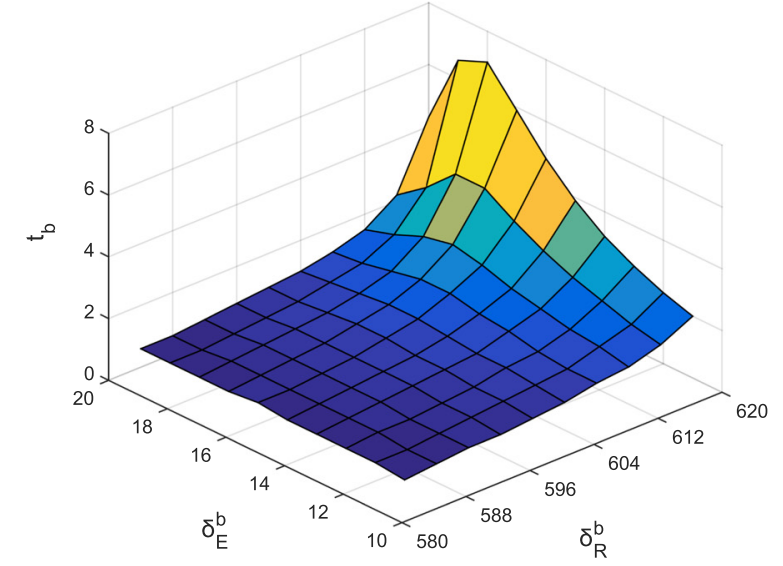

Fig. 13 Effect of the system parameters when initially the state is 0 . $\mathbf{a}$ Effects of $\xi$ on $g_{x}$. $\mathbf{b}$ Effects of $\omega$ on $g_{x}$. $\mathbf{c}$ Effects of $\delta_{E}^{b}, \delta_{R}^{b}$ on $\hat{t}_{b}$

\subsection{Investigation on the effect of system parameters}

In this subsection, we discuss the effect of parameters. Besides the parameters we discussed, the other parameters are set according to Table 2. Figure $12 \mathrm{a}-\mathrm{c}$ illustrates the simulation results when the initial beaconing result 
is 0 . Figure 12a shows the possible region of $f_{X}$ when $\omega=300 \mathrm{kbit}$ and $\xi$ changes from 8.5 to $12 \mathrm{~mJ}$. Figure $12 \mathrm{~b}$ illustrates the influence of $\omega$ on the objective function $f_{X}$ when $\xi=10 \mathrm{~mJ}$. Since $\xi$ affects the decreased energy consumption $f_{E}\left(t_{a}\right)$, and $\omega$ is related to the decreased data rate $f_{R}\left(t_{a}\right)$, the value of $f_{X}$ is affected by them. $\delta_{E}^{a}$ and $\delta_{R}^{a}$ are the thresholds when the initial beaconing result is 0 . $\delta_{E}^{a}$ is a predefined threshold reflecting the energy requirement, and $\delta_{R}^{a}$ is a predefined threshold reflecting the data rate requirement. Figure 12b shows the relationship between $\delta_{E}^{a}$, $\delta_{R}^{a}$, and $\hat{t}_{a}$. By adaptively adjusting the threshold $\delta_{E}^{a}$ and $\delta_{R}^{a}$, the roadside infrastructure can satisfy its various requirements. Specifically, if the roadside infrastructure prefers to work in an energy-saving mode, it can choose a long beaconing interval so as to save as much energy as possible. As shown in Fig. 12c, for a certain $\delta_{E}^{a}$, the optimal beaconing interval $\hat{t}_{a}$ increases with the increase of $\delta_{R}^{a}$. On the contrary, if the roadside infrastructure wants to disseminate more data, it can send the beacon packets more frequently. Figure $13 \mathrm{a}-\mathrm{c}$ illustrates the simulation results when the initial beaconing result is 1 . The investigation is similar.

\section{Conclusions}

In this paper, we propose an energy-efficient data dissemination strategy for roadside infrastructure in VCPS. We model the beaconing procedure in the data dissemination strategy as a Markov model. With obtaining the relationship between the beaconing interval and the expectation of the single-vehicle discovery time, we can calculate the maximum beaconing interval. By using the Sigmoid function, we can obtain the optimal beaconing interval to based on the satisfaction degree. The impact of the system parameters on the data dissemination strategy is investigated via extensive simulation study. Our future work involves extensive empirical investigations and analytical studies of the proposed approach.

\section{Competing interests}

The authors declare that they have no competing interests.

\section{Acknowledgements}

The authors would like to thank the support from the National Natural Science Foundation of China (Grant Nos. 61471028, 61371069, and 61272505).

Received: 31 December 2015 Accepted: 5 June 2016

Published online: 16 June 2016

\section{References}

1. AA Cardenas, S Amin, S Sastry, in Proc. IEEE ICDCS. Secure control: towards survivable cyber-physical systems, (2008), pp. 495-500. doi:10.1109/ICD CS.Workshops.2008.40

2. Y Huang, X Guan, Z Cai, T Ohtsuki, in 2013 IEEE International Conference on Communications (ICC). Multicast capacity analysis for social-proximity urban bus-assisted VANETs, (2013), pp. 6138-6142. doi:10.1109/ICC.2013. 6655586

3. J Shi, J Wan, H Yan, H Suo, in Proc. WCSP. A survey of cyber-physical systems, (2011), pp. 1-6. doi:10.1109/WCSP.2011.6096958

4. X Wang, L Guo, C Ai, J Li, Z Cai, in 8th International Conference, WASA 2013, Zhangjiajie, China, August 7-10, 2013. Proceedings. Wireless Algorithms,
Systems, and Applications (Springer Berlin Heidelberg, 2013), pp. 313-324. doi:10.1007/978-3-642-39701-1_26

5. X Li, X Yu, A Wagh, C Qiao, in Proc. IEEE INFOCOM. Human factors-aware service scheduling in vehicular cyber-physical systems, (2011), pp. 2174-2182. doi:10.1109/INFCOM.2011.5935030

6. K Liu, VCS Lee, JK-Y Ng, J Chen, SH Son, Temporal data dissemination in vehicular cyber x2013; physical systems. IEEE Transactions on Intelligent Transportation Systems. 15(6), 2419-2431 (2014). doi:10.1109/TITS.2014 2316006

7. X Zheng, Z Cai, J Li, H Gao, in Distributed Computing Systems (ICDCS), 2015 IEEE 35th International Conference On. An application-aware scheduling policy for real-time traffic, (2015), pp. 421-430. doi:10.1109/ICDCS.2015.50

8. D Jia, K Lu, J Wang, X Zhang, X Shen, A survey on platoon-based vehicular cyber-physical systems. IEEE Communications Surveys Tutorials. PP(99)، 1-1 (2015). doi:10.1109/COMST.2015.2410831

9. J Gozalvez, M Sepulcre, R Bauza, IEEE 802.11 p vehicle to infrastructure communications in urban environments. IEEE Communications Magazine. 50(5), 176-183 (2012). doi:10.1109/MCOM.2012.6194400

10. A Paier, R Tresch, A Alonso, D Smely, P Meckel, Y Zhou, N Czink, in Proc. IEEE ICC. Average downstream performance of measured IEEE $802.11 \mathrm{p}$ infrastructure-to-vehicle links, (2010), pp. 1-5. doi:10.1109/ICCW.2010. 5503934

11. Y Huang, M Chen, Z Cai, X Guan, T Ohtsuki, Y Zhang, in 2015 IEEE Global Communications Conference (GLOBECOM). Graph theory based capacity analysis for vehicular ad hoc networks, (2015), pp. 1-5. doi:10.1109/ GLOCOM.2015.7417561

12. Y Wang, J Zheng, N Mitton, in Proc. IEEE GLOBECOM. Delivery delay analysis for roadside unit deployment in intermittently connected VANETs, (2014), pp. 155-161. doi:10.1109/GLOCOM.2014.7036800

13. S Peirce, R Mauri, in Intell. Transp. Syst. Vehicle-infrastructure integration (VII) initiative benefit-cost analysis: pre-testing estimates (US DoT draft report, Washington, DC, 2007)

14. M Kohvakka, M Hannikainen, TD Hamalainen, in 2005 IEEE 16th International Symposium on Personal, Indoor and Mobile Radio Communications. Energy optimized beacon transmission rate in a wireless sensor network, vol. 2, (2005), pp. 1269-12732. doi:10.1109/PIMRC.2005.1651645

15. R Lasowski, C Linnhoff-Popien, Beaconing as a service: a novel service-oriented beaconing strategy for vehicular ad hoc networks. IEEE Communications Magazine. 50(10), 98-105 (2012). doi:10.1109/MCOM. 2012.6316782

16. A Daniel, DC Popescu, S Olariu, in Proc. IEE ICC. A study of beaconing mechanism for vehicle-to-infrastructure communications, (2012), pp. 7146-7150. doi:10.1109/ICC.2012.6364667

17. AA Hammad, GH Badawy, TD Todd, AA Sayegh, D Zhao, in Proc. IEEE GLOBECOM. Traffic scheduling for energy sustainable vehicular infrastructure, (2010), pp. 1-6. doi:10.1109/GLOCOM.2010.5683759

18. AA Hammad, TD Todd, G Karakostas, D Zhao, Downlink traffic scheduling in green vehicular roadside infrastructure. IEEE Transactions on Vehicular Technology. 62(3), 1289-1302 (2013). doi:10.1109/TVT.2012.2227071

19. Z Yan, B Li, X Zuo, T Gao, in Proc. ICCVE. Fair downlink traffic scheduling for energy sustainable vehicular roadside infrastructure, (2014), pp. 1092-1097. doi:10.1109/ICCVE.2014.7297519

20. S Mostofi, A Hammad, TD Todd, G Karakostas, in Proc. IEEE ICC. On/off sleep scheduling in energy efficient vehicular roadside infrastructure, (2013), pp. 6266-6271. doi:10.1109/ICC.2013.6655611

21. C Wen, J Zheng, in Proc. WCSP. An RSU on/off scheduling mechanism for energy efficiency in sparse vehicular networks, (2015), pp. 1-5. doi:10.1109/WCSP.2015.7341227

22. AA Hammad, TD Todd, G Karakostas, Variable bit rate transmission schedule generation in green vehicular roadside units. IEEE Transactions on Vehicular Technology. PP(99), 1-1 (2015). doi:10.1109/TVT.2015. 2410798

23. Y Yao, L Rao, X Liu, X Zhou, in Proc. IEEE INFOCOM. Delay analysis and study of IEEE 802.11p based DSRC safety communication in a highway environment, (2013), pp. 1591-1599. doi:10.1109/INFCOM.2013.6566955

24. S Bitam, A Mellouk, in Proc. IEEE VTC. Markov-history based modeling for realistic mobility of vehicles in VANETs, (2013), pp. 1-5. doi:10.1109/ VTCSpring.2013.6692628

25. X Chen, L Li, Y Zhang, A Markov model for headway/spacing distribution of road traffic. IEEE Transactions on Intelligent Transportation Systems. 11(4), 773-785 (2010). doi:10.1109/TITS.2010.2050141 